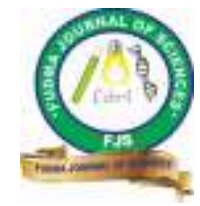

FUDMA Journal of Sciences (FJS)

ISSN online: $2616-1370$

ISSN print: 2645 - 2944

Vol. 4 No. 2, June, 2020, pp $523-531$

DOI: https://doi.org/10.33003/fjs-2020-0402-185

\title{
POSITIONS AND STABILITY OF LIBRATION POINTS IN THE RADIATING AND OBLATING BIGGER PRIMARY OF CIRCULAR RESTRICTED THREE-BODY PROBLEM
}

\author{
${ }^{* 1}$ Tyokyaa, K. R. and ${ }^{2}$ Atsue, $\mathbf{T}$. \\ ${ }^{1}$ Department of Mathematical Sciences, Faculty of Physical Sciences, Federal University Dutsin-Ma, Katsina State, Nigeria \\ ${ }^{2}$ Department of Physics, Faculty of Physical Sciences, Federal University Dutsin-Ma, Katsina State, Nigeria
}

${ }^{*}$ Corresponding author's email: rtkanshio6@gmail.com

\begin{abstract}
This paper investigates the positions and stability of libration points in the framework of the circular restricted three-body problem for the systems: Luyten726-8 and HD98800. The position of the third body is contained in the $x Z$-plane direction above and below the oblate bigger and smaller primaries. It is observed that presence of radiations and oblateness of the primary affect the stability of the libration points. Considering the range of stability and instability, that is $0<\mu \leq \mu_{c}$ and $\mu_{c}<\mu \leq \frac{1}{2}$, the libration points are respectively stable and unstable for HD98800 and Luyten 762-8 systems. Our results show that, all the roots are real, and for each set of values, there exist at least a positive real part and hence in the Lyapunov sense, the stability of the libration points are unstable for the systems HD98800 and Luyten 762-8.
\end{abstract}

Keywords: Luyten726-8, Libration points, Radiations, Oblateness

\section{INTRODUCTION}

The masses of the planets compared to its approximate circular motion around the Sun and the small masses of asteroids and the satellites of planets constitute the restricted problem. The restricted three-body problem (R3BP) assumes the participating bodies are spherical having circular orbits. Researchers observed that the motion of two spherical massive bodies (primaries) in circular orbits influenced the third mass-less body under their mutual gravitational attraction, however the third mass-less body has no gravitational influence on the two spherical massive bodies. A system of this nature contains three collinear equilibrium points whose lines join the primaries and two equilibrium points forming the equilateral triangles with the primaries making five co-planar equilibrium points (Singh and Ishwar 1999, Singh and Begha 2011, Singh and Leke 2014).

The equations of motion expressed in a rotating coordinate system contain coriolis terms that stabilized the triangular equilibrium points. However, triangular equilibrium solution becomes unstable in the absence of the coriolis force, thus the exponential terms with real characteristic exponents replaced the oscillatory solution of the linearized equations of motion (Wintner 1941). Another researcher observed that in the presence of a small perturbation on the triangular points, the collinear points became unstable while that of triangular points are stabilized by the coriolis force (Szebehely 1967). The change $\varepsilon$ in the coriolis force is related to the critical mass parameter $\mu_{c}$ value by the expression $\mu_{c}=\mu_{0}+\frac{16 \varepsilon}{\sqrt[3]{69}}$

Triangular equilibrium points' stability of the photogravitational R3BP has been studied for the case in which the source of radiation and oblate spheroid is a massive primary (Sun) by
Sharma in 1982. He observed that, in the linear sense there exist a collinear retrograde elliptical periodic orbits around the triangular points, whereas for the mass parameter the triangular points have long or short-periodic retrograde elliptical orbits leading to increase in oblateness and radiation force as the value of the critical mass parameter decreases.

The stability of equilibrium points in the R3BP under the influence of small perturbations in the Coriolis and centrifugal forces was examined together with the effects of oblateness and radiation pressure of the primaries. Observation showed that the collinear points were generally unstable, while the triangular points were conditionally stable for $0<\mu<\mu_{c}$ and unstable for $\mu_{c}<\mu \leq \frac{1}{2}$ (AbdulRaheem and Singh 2006). This has been confirmed by many researchers with further observation that the presence of the centrifugal force, radiations and oblateness of the primary affect the stability of the libration points while its stability is regained with the presence of Coriolis force. (Douskos and Markellos 2006, Singh and Umar, 2012, 2013, Singh and Leke, 2013, Singh and Amuda, 2015, Singh and Tyokyaa 2016, 2017). Abouelmagd and El-Shaboury (2012) observed that the collinear points of the linear stability of libration points in the axisymmetric R3BP with radiating primaries remain unstable.

Others researchers examined the combine effects of radiation and oblateness up to zonal harmonics $\left(J_{4}\right)$ of both primaries, considering additional gravitational potential arising from the circular cluster of material points when an infinitesimal body moved under the frame-work of circular R3BP. The locations of the triangular points and their linear stability are found to be affected by the oblateness up to $J_{4}$ of the bigger primary in the 
planar circular R3BP (Singh and Taura, 2013; Abouelmagd, 2012).

For the position and velocity sensitivities at the triangular libration points, Hassan et al. (2013) observed that when the bigger primary oblates in $\mathrm{R} 3 \mathrm{BP}$, the critical mass $\left(\mu_{c}\right)$ value reduced leading to a reduction in the range of stability of the equilateral triangular libration points while the oblateness parameter increased and hence there was an increase in the order of commensurability.

In the literature search, little or nothing has been considered on the perturbation of the binary systems Luyten726-8 and
HD98800 on the stability of libration points. Our work therefore extends that of Hassan and coworkers to include the radiation pressure of the bigger primary for the aforementioned binary systems.

The study is presented in the sections as follows: section 2 provides the equations of motion; section 3 locates the libration points; section 4 presents the mathematical formulation for the critical mass value and section 5 gives results and discussion of the problem. Lastly, the conclusions are provided in section 6 .

\section{Equations of motion}

The equations of motion of the third body moving in the framework of the circular restricted three-body problem of the two primaries are given as thus;

$\ddot{x}-2 n \dot{y}=\Omega_{x}, \quad \ddot{y}-2 n \dot{x}=\Omega_{y}$

with the force function

$\Omega=\frac{n^{2}}{2}\left(x^{2}+y^{2}\right)+\frac{(1-\mu) q_{1}}{r_{1}}+\frac{\mu q_{2}}{r_{2}}+\frac{3 I_{1} q_{1}}{2 r_{1}{ }^{3}}$

The mean motion, $n$, is given as

$n^{2}=1+\frac{3}{2} I_{1}$

$r_{1}{ }^{2}=(x+\mu)^{2}+y^{2}, r_{2}{ }^{2}=(x+\mu-1)^{2}+y^{2}$

\section{Location of libration points}

The libration points are the solutions of the equations $\Omega_{x}=\Omega_{y}=0$. Considering equation (2) we have;

$\Omega_{x}=x n^{2}-\frac{(1-\mu)(x+\mu) q_{1}}{r_{1}{ }^{3}}-\frac{\mu(x+\mu-1) q_{2}}{r_{2}{ }^{3}}-\frac{9(x+\mu) I_{1} q_{1}}{2 r_{1}{ }^{5}}$

$\Omega_{y}=y\left[n^{2}-\frac{(1-\mu) q_{1}}{r_{1}{ }^{3}}-\frac{\mu q_{2}}{r_{2}{ }^{3}}-\frac{9 I_{1} q_{1}}{2 r_{1}{ }^{5}}\right]$

For the triangular libration points, the solution of equations $5 \& 6$ will be zero i.e

$x n^{2}-\frac{(1-\mu)(x+\mu) q_{1}}{r_{1}{ }^{3}}-\frac{\mu(x+\mu-1) q_{2}}{r_{2}{ }^{3}}-\frac{9(x+\mu) I_{1} q_{1}}{2 r_{1}{ }^{5}}=0$

$y\left[n^{2}-\frac{(1-\mu) q_{1}}{r_{1}{ }^{3}}-\frac{\mu q_{2}}{r_{2}{ }^{3}}-\frac{9 I_{1} q_{1}}{2 r_{1}{ }^{5}}\right]=0$

The positions of the libration points are obtained from the solutions of equation $7 \& 8$ with $y \neq 0$ and $x=0$; we obtain

$n^{2}=\frac{q_{1}}{r_{1}{ }^{3}}+\frac{9 I_{1} q_{1}}{2(1-\mu) r_{1}{ }^{5}}=0, n^{2}=\frac{q_{2}}{r_{2}{ }^{3}}$

When oblateness and radiation of the bigger primary is absent, then the equations of (9) becomes $n^{2}=\frac{1}{r_{1}{ }^{3}}$ which implies that $r_{1}^{3}=\frac{1}{n^{2}}$ and $n^{2}=\frac{1}{r_{2}{ }^{3}}$ which implies that $r_{2}{ }^{3}=\frac{1}{n^{2}}$ respectively.

Introducing oblateness and radiation, the values change slightly by $\varepsilon_{1}, \varepsilon_{2}$ (say) so that

$r_{1}=\frac{1}{n^{\frac{2}{3}}}+\varepsilon_{1}, r_{2}=\frac{1}{n^{\frac{2}{3}}}+\varepsilon_{2}, \varepsilon_{1}, \varepsilon_{2}<<1$

Given that, the mean motion

$n^{2}=1+\frac{3}{2} I_{1}$, and considering $I_{1}=0$ for oblateness, we have

$n^{2}=1$

Which means that $n^{\frac{2}{3}}=\left(n^{2}\right)^{\frac{1}{3}}=1^{\frac{2}{3}}=1$

Substituting (12) into (10) we have

$r_{1}=1+\varepsilon_{1}, r_{2}=1+\varepsilon_{2}, \varepsilon_{1}, \varepsilon_{2}<<1$

Considering equations 10 to 13 we have;

$n^{2}=q_{1}\left(1+3 \varepsilon_{1}\right)+\frac{9 I_{1} q_{1}}{2(1-\mu)}\left(1+5 \varepsilon_{1}\right)$

Solving further and neglecting higher powers of $I_{1}, q_{1}, q_{2}$ and their products, the positions of the libration points are given as thus; 
$x=\frac{1}{2}-\mu+\varepsilon_{1}+\varepsilon_{2}$

$y=\left(\frac{3}{4}+\varepsilon_{1}+\varepsilon_{2}\right)^{\frac{1}{2}}$

where;

$\varepsilon_{1}=\frac{1}{\left(6 q_{1}(1-\mu)+45 q_{1} I_{1}\right)}\left(2(1-\mu)+3 I_{1}(1-\mu)-2 q_{1}(1-\mu)-9 I_{1} q_{1}\right)$

$\varepsilon_{2}=\frac{1}{\left(6 \mu q_{2}\right)}\left(2 \mu+3 \mu I_{1}-2 \mu q_{2}\right)$

\section{Mathematical Formulation for the Critical mass value ( $\left.\boldsymbol{\mu}_{c}\right)$}

To formulate the equation for the critical mass value denoted by $\mu_{c}$, we obtain the characteristics equation which is derived from the equations of the third body moving in the gravitational field of the two primaries as thus;

$\ddot{x}-2 n \dot{y}=\frac{\partial \Omega}{\partial x}$,

$\ddot{y}+2 n \dot{x}=\frac{\partial \Omega}{\partial y}$

Let the positions of the motion of the third body near the libration points be denoted by $\left(x_{0}, y_{0}\right)$ and then assume the small displacements in $\left(x_{0}, y_{0}\right)$ to be $(\alpha, \beta)$ such that $x=x_{0}+\alpha$ and $y=y_{0}+\beta$

Taking the derivatives, we obtain,

$\dot{x}=\dot{\alpha}, \ddot{x}=\ddot{\alpha}$

$\dot{y}=\dot{\beta}, \ddot{y}=\ddot{\beta}$

Considering equations (18) into (17) we have;

$\ddot{\alpha}-2 n \dot{\beta}=\left(\Omega_{x x}^{0}\right) \alpha+\left(\Omega_{x y}^{0}\right) \beta$

$\ddot{\beta}+2 n \dot{\alpha}=\left(\Omega_{x y}^{0}\right) \alpha+\left(\Omega_{y y}^{0}\right) \beta$

Now, let

$\alpha=k_{1} e^{\lambda t}$ likewise $\beta=k_{2} e^{\lambda t}$

Considering equations 18 to 20 we obtain the characteristic equation as;

$\Lambda^{2}+P \Lambda+Q=0$

Where

$\lambda^{2}=\Lambda, \quad P=4 n^{2}-\Omega_{x x}^{0}-\Omega_{y y}^{0}, Q=\Omega_{x x}^{0} \Omega_{y y}^{0}-\left(\Omega_{x y}^{0}\right)^{2}$

Solving for the value of $P \& Q$ in equation 21 we have

$\Omega_{x x}^{0}=n^{2}-\frac{(1-\mu) q_{1}}{\left(1+3 \varepsilon_{1}\right)}-\frac{\mu q_{2}}{\left(1+3 \varepsilon_{2}\right)}-\frac{9 I_{1} q_{1}}{2\left(1+5 \varepsilon_{1}\right)}+\frac{3(1-\mu)(x+\mu)^{2} q_{1}}{\left(1+5 \varepsilon_{1}\right)}+\frac{3 \mu(x+\mu-1)^{2} q_{2}}{\left(1+5 \varepsilon_{2}\right)}+\frac{45(x+\mu)^{2} I_{1} q_{1}}{2\left(1+7 \varepsilon_{1}\right)}$

$\Omega_{y y}^{0}=y^{2}\left(\frac{3(1-\mu) q_{1}}{\left(1+5 \varepsilon_{1}\right)}+\frac{3 \mu q_{2}}{\left(1+5 \varepsilon_{2}\right)}+\frac{45 I_{1} q_{1}}{2\left(1+7 \varepsilon_{1}\right)}\right)$

$\left(\Omega_{x y}^{0}\right)^{2}=0$

$P=3 n^{2}+\frac{(1-\mu) q_{1}}{\left(1+3 \varepsilon_{1}\right)}+\frac{\mu q_{2}}{\left(1+3 \varepsilon_{2}\right)}+\frac{9 I_{1} q_{1}}{2\left(1+5 \varepsilon_{1}\right)}-\frac{3(1-\mu)(x+\mu)^{2} q_{1}}{\left(1+5 \varepsilon_{1}\right)}-\frac{3 \mu(x+\mu-1)^{2} q_{2}}{\left(1+5 \varepsilon_{2}\right)}-\frac{45(x+\mu)^{2} I_{1} q_{1}}{2\left(1+7 \varepsilon_{1}\right)}-y^{2}\left(\frac{3(1-\mu) q_{1}}{\left(1+5 \varepsilon_{1}\right)}+\frac{3 \mu q_{2}}{\left(1+5 \varepsilon_{2}\right)}+\frac{45 I_{1} q_{1}}{2\left(1+7 \varepsilon_{1}\right)}\right)$

$Q=n^{2} y^{2}\left(\frac{3(1-\mu) q_{1}}{\left(1+5 \varepsilon_{1}\right)}+\frac{3 \mu q_{2}}{\left(1+5 \varepsilon_{2}\right)}+\frac{45 I_{1} q_{1}}{2\left(1+7 \varepsilon_{1}\right)}\right)$

Making use of equations $23 \& 24$ in equation 21 yields;

$\Lambda^{2}+\left(3 n^{2}+\frac{(1-\mu) q_{1}}{\left(1+3 \varepsilon_{1}\right)}+\frac{\mu q_{2}}{\left(1+3 \varepsilon_{2}\right)}+\frac{9 I_{1} q_{1}}{2\left(1+5 \varepsilon_{1}\right)}-\frac{3(1-\mu)(x+\mu)^{2} q_{1}}{\left(1+5 \varepsilon_{1}\right)}-\frac{3 \mu(x+\mu-1)^{2} q_{2}}{\left(1+5 \varepsilon_{2}\right)}-\frac{45(x+\mu)^{2} I_{1} q_{1}}{2\left(1+7 \varepsilon_{1}\right)}-y^{2}\left(\frac{3(1-\mu) q_{1}}{\left(1+5 \varepsilon_{1}\right)}+\frac{3 \mu q_{2}}{\left(1+5 \varepsilon_{2}\right)}+\right.\right.$

$\left.\left.\frac{45 I_{1} q_{1}}{2\left(1+7 \varepsilon_{1}\right)}\right)\right) \Lambda+n^{2} y^{2}\left(\frac{3(1-\mu) q_{1}}{\left(1+5 \varepsilon_{1}\right)}+\frac{3 \mu q_{2}}{\left(1+5 \varepsilon_{2}\right)}+\frac{45 I_{1} q_{1}}{2\left(1+7 \varepsilon_{1}\right)}\right)=0$

To determine the value of the critical mass, we solve for the discriminant of equation (25). The discriminant of a quadratic equation $a x^{2}+b x+c=0$ is given by $b^{2}-4 a c$. Now, at the critical point, we are interested in a quadratic equation where the discriminant is equal to zero that is $b^{2}-4 a c=0$. That is;

$b^{2}-4 a c=9 n^{4}+\frac{6 n^{2}(1-\mu) q_{1}}{\left(1+3 \varepsilon_{1}\right)}+\frac{6 n^{2} \mu q_{2}}{\left(1+3 \varepsilon_{2}\right)}+\frac{54 n^{2} I_{1} q_{1}}{2\left(1+5 \varepsilon_{1}\right)}-\frac{18 n^{2}(1-\mu)(x+\mu)^{2} q_{1}}{\left(1+5 \varepsilon_{1}\right)}-\frac{18 n^{2} \mu(x+\mu-1)^{2} q_{2}}{\left(1+5 \varepsilon_{2}\right)}-\frac{270 n^{2}(x+\mu)^{2} I_{1} q_{1}}{2\left(1+7 \varepsilon_{1}\right)}-$

$10 n^{2} y^{2}\left(\frac{3(1-\mu) q_{1}}{\left(1+5 \varepsilon_{1}\right)}+\frac{3 \mu q_{2}}{\left(1+5 \varepsilon_{2}\right)}+\frac{45 I_{1} q_{1}}{2\left(1+7 \varepsilon_{1}\right)}\right)$

But

$b^{2}-4 a c=0$ 
That is;

$9 n^{4}+\left(\frac{6(1-\mu) q_{1}}{\left(1+3 \varepsilon_{1}\right)}+\frac{6 \mu q_{2}}{\left(1+3 \varepsilon_{2}\right)}+\frac{54 I_{1} q_{1}}{2\left(1+5 \varepsilon_{1}\right)}-\frac{18(1-\mu)(x+\mu)^{2} q_{1}}{\left(1+5 \varepsilon_{1}\right)}-\frac{18 \mu(x+\mu-1)^{2} q_{2}}{\left(1+5 \varepsilon_{2}\right)}-\frac{270(x+\mu)^{2} I_{1} q_{1}}{2\left(1+7 \varepsilon_{1}\right)}-10 y^{2}\left(\frac{3(1-\mu) q_{1}}{\left(1+5 \varepsilon_{1}\right)}+\frac{3 \mu q_{2}}{\left(1+5 \varepsilon_{2}\right)}+\right.\right.$

$\left.\left.\frac{45 I_{1} q_{1}}{2\left(1+7 \varepsilon_{1}\right)}\right)\right) n^{2}=0$

Considering equation (26) as a quadratic equation we have;

$9 \lambda^{2}+R \lambda=0$

Where;

$n^{2}=\lambda$

$R=\frac{6(1-\mu) q_{1}}{\left(1+3 \varepsilon_{1}\right)}+\frac{6 \mu q_{2}}{\left(1+3 \varepsilon_{2}\right)}+\frac{54 I_{1} q_{1}}{2\left(1+5 \varepsilon_{1}\right)}-\frac{18(1-\mu)(x+\mu)^{2} q_{1}}{\left(1+5 \varepsilon_{1}\right)}-\frac{18 \mu(x+\mu-1)^{2} q_{2}}{\left(1+5 \varepsilon_{2}\right)}-\frac{270(x+\mu)^{2} I_{1} q_{1}}{2\left(1+7 \varepsilon_{1}\right)}-10 y^{2}\left(\frac{3(1-\mu) q_{1}}{\left(1+5 \varepsilon_{1}\right)}+\frac{3 \mu q_{2}}{\left(1+5 \varepsilon_{2}\right)}+\frac{45 I_{1} q_{1}}{2\left(1+7 \varepsilon_{1}\right)}\right)$

Solving equation (27) using the general formula of the quadratic equation given by $\lambda=\frac{-b \pm \sqrt{b^{2}-4 a c}}{2 a}$ ) we have;

$a=9, b=R, c=0$

$\lambda=\frac{-R+R}{18}$ or $\lambda=\frac{-R-R}{18}$

$\lambda=0$ or $\lambda=\frac{-R-R}{18}$

Where;

$R=\frac{6(1-\mu) q_{1}}{\left(1+3 \varepsilon_{1}\right)}+\frac{6 \mu q_{2}}{\left(1+3 \varepsilon_{2}\right)}+\frac{54 I_{1} q_{1}}{2\left(1+5 \varepsilon_{1}\right)}-\frac{18(1-\mu)(x+\mu)^{2} q_{1}}{\left(1+5 \varepsilon_{1}\right)}-\frac{18 \mu(x+\mu-1)^{2} q_{2}}{\left(1+5 \varepsilon_{2}\right)}-\frac{270(x+\mu)^{2} I_{1} q_{1}}{2\left(1+7 \varepsilon_{1}\right)}-10 y^{2}\left(\frac{3(1-\mu) q_{1}}{\left(1+5 \varepsilon_{1}\right)}+\frac{3 \mu q_{2}}{\left(1+5 \varepsilon_{2}\right)}+\frac{45 I_{1} q_{1}}{2\left(1+7 \varepsilon_{1}\right)}\right)$

$x=\frac{1}{2}-\mu+\varepsilon_{1}+\varepsilon_{2}$

$y^{2}=\frac{3}{4}+\varepsilon_{1}+\varepsilon_{2}$

$\varepsilon_{1}=\frac{1}{\left(6 q_{1}(1-\mu)+45 q_{1} I_{1}\right)}\left(2(1-\mu)+3 I_{1}(1-\mu)-2 q_{1}(1-\mu)-9 I_{1} q_{1}\right)$

$\varepsilon_{2}=\frac{1}{\left(6 \mu q_{2}\right)}\left(2 \mu+3 \mu I_{1}-2 \mu q_{2}\right)$

\section{RESULTS AND DISCUSSION}

Considering equations (15) and (16), we generate numerical values for the locations of the libration points. Using equation (28) we established numerically the critical mass values of the stated problem. We compute numerically the roots of the quadratic equation of order 4 in equation (26) to ascertain the stability nature for the libration points.
These computations in the aforementioned equations are done with the help of a software package MATHEMATICA for the systems Luyten 762-8 and HD98800. The effects of the parameters on the positions and stability of the libration points are shown numerically in Tables 1-4 and graphically in Figures $1-6$. 
Table 1: Effects of radiations and oblateness on the positions of the libration points for the systems: Luyten 762-8 and HD98800.

\begin{tabular}{|c|c|c|c|c|c|c|}
\hline \multirow[t]{2}{*}{ Binary Systems } & \multirow[t]{2}{*}{ Mass ratio $(\mu)$} & \multicolumn{2}{|c|}{ Radiations } & \multirow[t]{2}{*}{$I_{1}$} & \multicolumn{2}{|c|}{ Oblateness } \\
\hline & & $q_{1}$ & $q_{2}$ & & $L_{4}$ & $L_{5}$ \\
\hline Luyten762-8 & 0.49550 & 0.00006 & 0.00004 & $\begin{array}{c}-0.006 \\
-0.005 \\
-0.004 \\
-0.003 \\
-0.002 \\
-0.001 \\
0.00 \\
0.001 \\
0.002 \\
0.003 \\
0.004 \\
0.005 \\
0.006\end{array}$ & $\begin{array}{c}-2213.61 \\
-2314.19 \\
-2411.98 \\
-2507.11 \\
-2599.71 \\
-2689.9 \\
-2777.77 \\
-2863.44 \\
-2946.99 \\
-3028.53 \\
-3108.12 \\
-3185.86 \\
-3261.82\end{array}$ & $\begin{array}{c}14303.1 \\
14227.6 \\
14154.8 \\
14084.6 \\
14017 \\
13951.8 \\
13889 \\
13828.3 \\
13769.8 \\
13713.2 \\
13658.6 \\
13605.9 \\
13554.9\end{array}$ \\
\hline HD98800 & 0.45344 & 0.405 & 0.205 & $\begin{array}{c}-0.006 \\
-0.005 \\
-0.004 \\
-0.003 \\
-0.002 \\
-0.001 \\
0.00 \\
0.001 \\
0.002 \\
0.003 \\
0.004 \\
0.005 \\
0.006\end{array}$ & $\begin{array}{l}-0.687968 \\
-0.700036 \\
-0.711824 \\
-0.723345 \\
-0.734609 \\
-0.745628 \\
-0.756411 \\
-0.766968 \\
-0.777309 \\
-0.787441 \\
-0.797373 \\
-0.807113 \\
-0.816668\end{array}$ & $\begin{array}{c}2.57157 \\
2.56438 \\
2.55747 \\
2.55083 \\
2.54444 \\
2.5383 \\
2.53239 \\
2.52672 \\
2.52125 \\
2.5160 \\
2.51094 \\
2.50608 \\
2.50141\end{array}$ \\
\hline
\end{tabular}

Table 2: Effects of radiations and oblateness on the locations and the Critical Mass Value $\left(\mu_{c}\right)$ of the libration points for the systems: Luyten 762-8 and HD98800.

\begin{tabular}{|c|c|c|c|c|c|c|c|}
\hline \multirow{2}{*}{$\begin{array}{c}\text { Binary } \\
\text { Systems } \\
\end{array}$} & \multirow{2}{*}{$\begin{array}{c}\text { Mass ratio } \\
(\mu)\end{array}$} & \multicolumn{2}{|c|}{ Radiations } & \multirow[t]{2}{*}{$\overline{I I I_{1}}$} & \multicolumn{2}{|c|}{ Locations } & \multirow{2}{*}{$\begin{array}{c}\text { Critical Mass Value } \\
\left(\mu_{c}\right)\end{array}$} \\
\hline & & $q_{1}$ & $q_{2}$ & & $L_{4}$ & $L_{5}$ & \\
\hline Luyten 762-8 & 0.49550 & 0.00006 & 0.00004 & $\begin{array}{c}-0.006 \\
-0.005 \\
-0.004 \\
-0.003 \\
-0.002 \\
-0.001 \\
0.00 \\
0.001 \\
0.002 \\
0.003 \\
0.004 \\
0.005 \\
0.006\end{array}$ & $\begin{array}{c}-2213.61 \\
-2314.19 \\
-2411.98 \\
-2507.11 \\
-2599.71 \\
-2689.9 \\
-2777.77 \\
-2863.44 \\
-2946.99 \\
-3028.53 \\
-3108.12 \\
-3185.86 \\
-3261.82\end{array}$ & $\begin{array}{c}14303.1 \\
14227.6 \\
14154.8 \\
14084.6 \\
14017 \\
13951.8 \\
13889 \\
13828.3 \\
13769.8 \\
13713.2 \\
13658.6 \\
13605.9 \\
13554.9\end{array}$ & $\begin{array}{c}0.0139598 \\
0.015508 \\
0.0171228 \\
0.0188031 \\
0.0205479 \\
0.0223561 \\
0.024227 \\
0.0261597 \\
0.0281537 \\
0.0302083 \\
0.0323231 \\
0.0344975 \\
0.0367312\end{array}$ \\
\hline HD98800 & 0.45344 & 0.405 & 0.205 & $\begin{array}{c}-0.006 \\
-0.005 \\
-0.004 \\
-0.003 \\
-0.002 \\
-0.001 \\
0.00 \\
0.001 \\
0.002 \\
0.003 \\
0.004 \\
0.005 \\
0.006\end{array}$ & $\begin{array}{l}-0.687968 \\
-0.700036 \\
-0.711824 \\
-0.723345 \\
-0.734609 \\
-0.745628 \\
-0.756411 \\
-0.766968 \\
-0.777309 \\
-0.787441 \\
-0.797373 \\
-0.807113 \\
-0.816668\end{array}$ & $\begin{array}{c}2.57157 \\
2.56438 \\
2.55747 \\
2.55083 \\
2.54444 \\
2.5383 \\
2.53239 \\
2.52672 \\
2.52125 \\
2.5160 \\
2.51094 \\
2.50608 \\
2.50141\end{array}$ & $\begin{array}{l}0.563214 \\
0.573690 \\
0.584333 \\
0.595142 \\
0.606118 \\
0.617261 \\
0.628571 \\
0.640048 \\
0.651694 \\
0.663507 \\
0.675489 \\
0.687640 \\
0.699960\end{array}$ \\
\hline
\end{tabular}


Table 3: Stability and locations of libration points for Luyten762-8 for $\mu=0.49550, q_{1}=0.00006$, and $q_{2}=0.00004$

\begin{tabular}{ccccc}
\hline \hline $\boldsymbol{I}_{\mathbf{1}}$ & \multicolumn{2}{c}{ Locations } & \multicolumn{2}{c}{ Stability of Libration points } \\
\cline { 2 - 5 } & $\boldsymbol{L}_{\mathbf{4}}$ & $\boldsymbol{L}_{\mathbf{5}}$ & $\pm \lambda_{1,2}$ & $\pm \lambda_{3,4}$ \\
\hline \hline-0.006 & -2213.61 & 14303.1 & \pm 0.00 & \pm 0.836636 \\
-0.005 & -2314.19 & 14227.6 & \pm 0.00 & \pm 0.124531 \\
-0.004 & -2411.98 & 14154.8 & \pm 0.00 & \pm 0.130854 \\
-0.003 & -2507.11 & 14084.6 & \pm 0.00 & \pm 0.137125 \\
-0.002 & -2599.71 & 14017 & \pm 0.00 & \pm 0.143345 \\
-0.001 & -2689.9 & 13951.8 & \pm 0.00 & \pm 0.155650 \\
0.00 & -2777.77 & 13889 & \pm 0.00 & \pm 0.161740 \\
0.001 & -2863.44 & 13828.3 & \pm 0.00 & \pm 0.167791 \\
0.002 & -2946.99 & 13769.8 & \pm 0.00 & \pm 0.173805 \\
0.003 & -3028.53 & 13713.2 & \pm 0.00 & \pm 0.179860 \\
0.004 & -3108.12 & 13658.6 & \pm 0.00 & \pm 0.185735 \\
0.005 & -3185.86 & 13605.9 & \pm 0.00 & \pm 0.191654 \\
0.006 & -3261.82 & 13554.9 & \\
\hline \hline
\end{tabular}

Table 4: Stability and locations of libration points for HD98800 for $\mu=0.45344, q_{1}=0.405$, and $q_{2}=0.205$

\begin{tabular}{ccccc}
\hline \hline $\boldsymbol{I}_{\mathbf{1}}$ & \multicolumn{2}{c}{ Locations } & \multicolumn{2}{c}{ Stability of Libration points } \\
\cline { 2 - 5 } & $\boldsymbol{L}_{\mathbf{4}}$ & $\boldsymbol{L}_{\mathbf{5}}$ & $\pm \lambda_{1,2}$ & $\pm \lambda_{3,4}$ \\
\hline \hline-0.006 & -0.687968 & 2.57157 & \pm 0.00 & \pm 0.750476 \\
-0.005 & -0.700036 & 2.56438 & \pm 0.00 & \pm 0.757423 \\
-0.004 & -0.711824 & 2.55747 & \pm 0.00 & \pm 0.764417 \\
-0.003 & -0.723345 & 2.55083 & \pm 0.00 & \pm 0.771455 \\
-0.002 & -0.734609 & 2.54444 & \pm 0.00 & \pm 0.778536 \\
-0.001 & -0.745628 & 2.5383 & \pm 0.00 & \pm 0.792825 \\
0.00 & -0.756411 & 2.53239 & \pm 0.800030 \\
0.001 & -0.766968 & 2.52672 & \pm 0.00 & \pm 0.807275 \\
0.002 & -0.777309 & 2.52125 & \pm 0.00 & \pm 0.814559 \\
0.003 & -0.787441 & 2.5160 & \pm 0.00 & \pm 0.821881 \\
0.004 & -0.797373 & 2.51094 & \pm 0.00 & \pm 0.829240 \\
0.005 & -0.807113 & 2.50608 & \pm 0.00 & \pm 0.836636 \\
0.006 & -0.816668 & 2.50141 & \\
\hline \hline
\end{tabular}

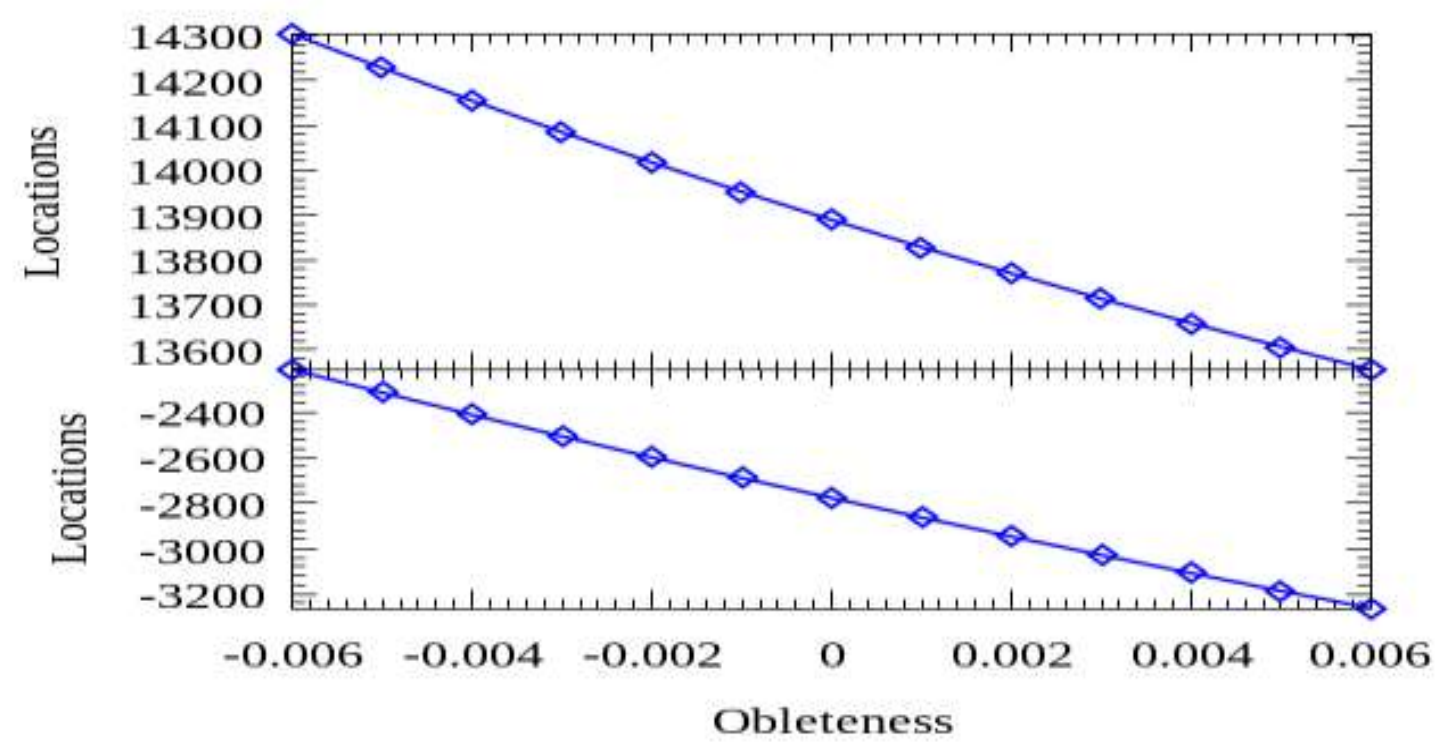

Figure 1: Effects of oblateness on $L_{4,5}$ for Luyten $762-8$ system with $\mu=0.49550, q_{1}=0.00006$, and $q_{2}=0.00004$ 


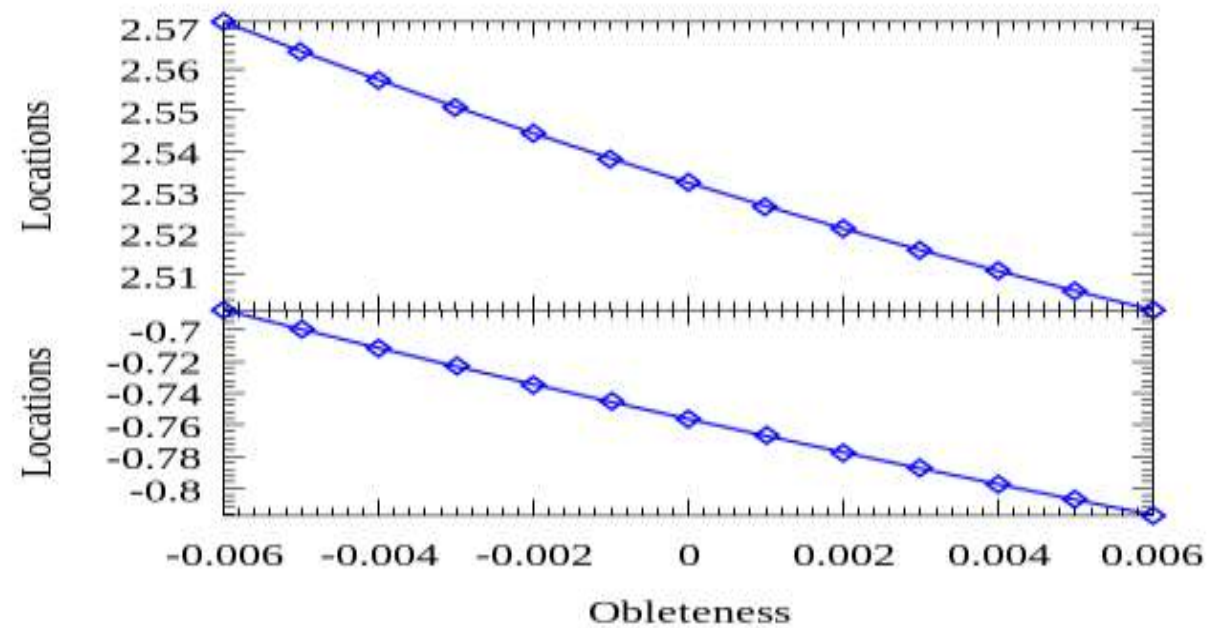

Figure 2: Effects of oblateness on $L_{4,5}$ for HD98800 system with $\mu=0.45344, q_{1}=0.405$, and $q_{2}=0.205$

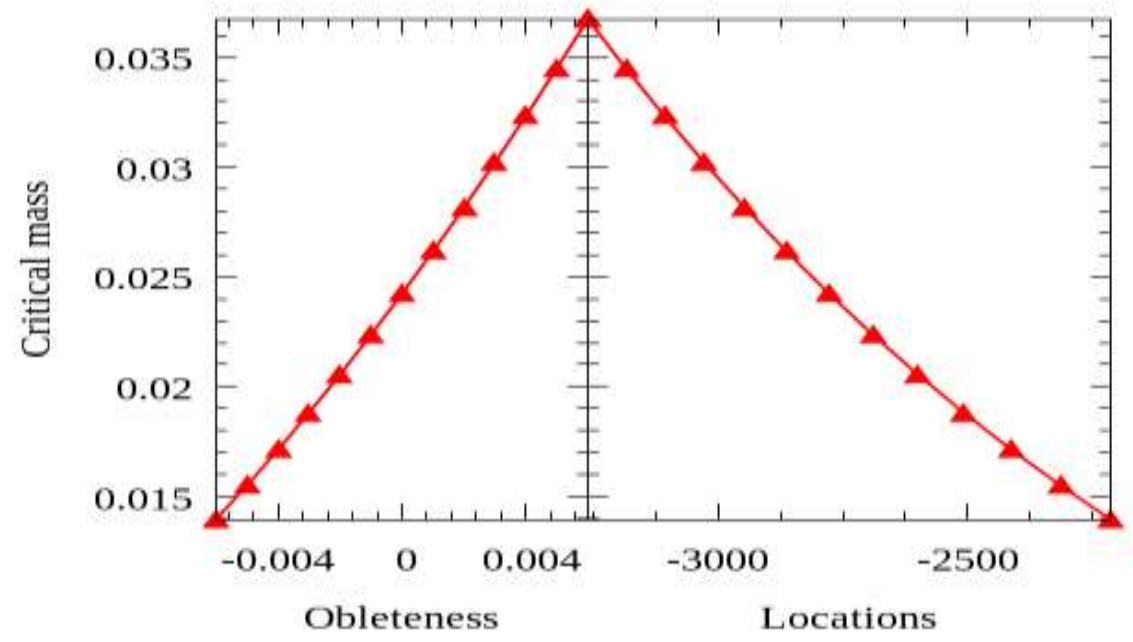

Figure 3: Effects of oblateness and $L_{4}$ on the critical mass value for Luyten762-8 system with $\mu=0.49550, q_{1}=0.00006$, and $q_{2}=0.00004$

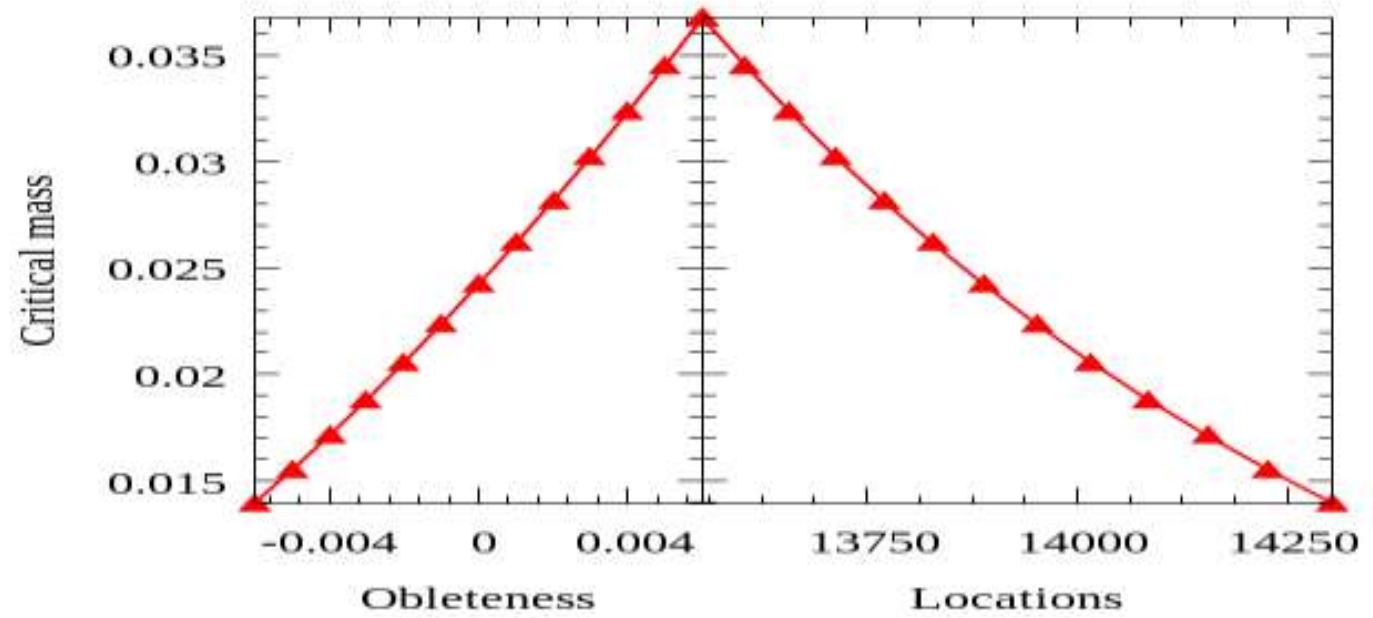

Figure 4: Effects of oblateness and $L_{5}$ on the critical mass value for Luyten762-8 system with $\mu=0.49550, q_{1}=0.00006$, and $q_{2}=0.00004$ 


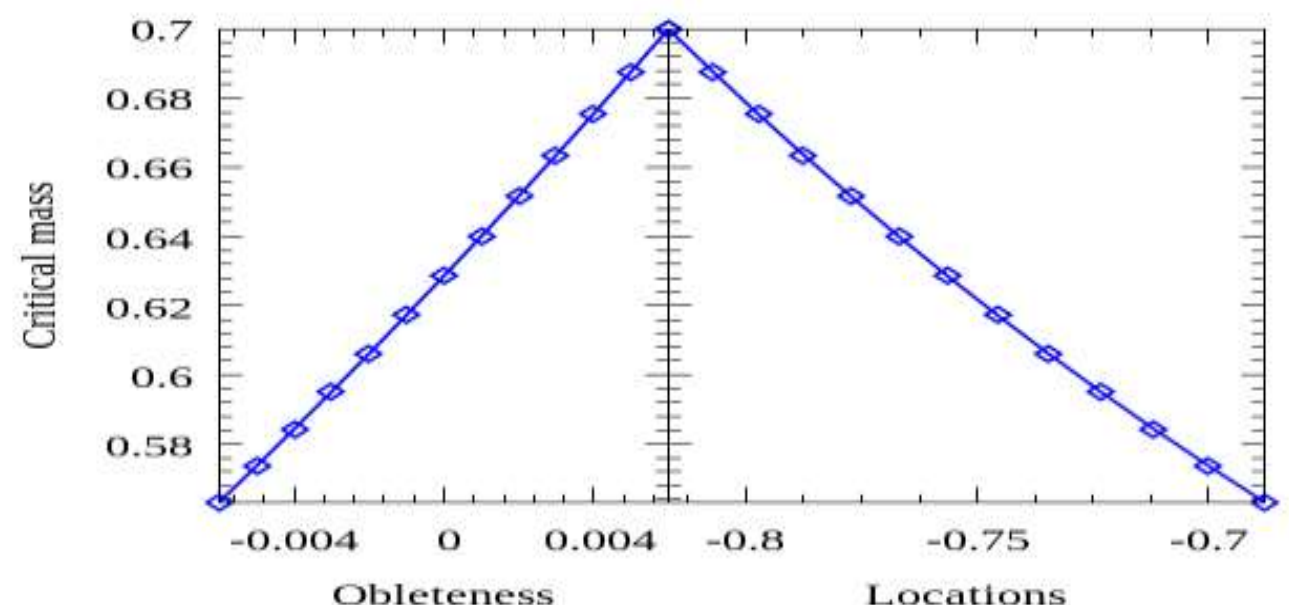

Figure 5: Effects of oblateness and $L_{4}$ on the critical mass value for HD98800 system with $\mu=0.45344, q_{1}=0.405$, and $q_{2}=$ 0.205

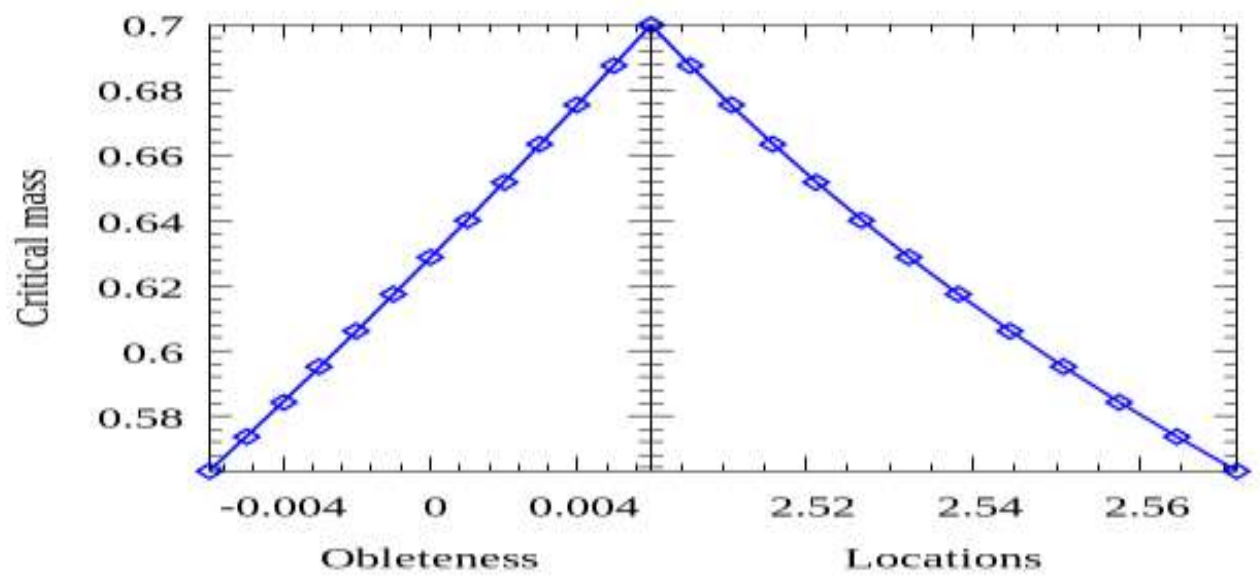

Figure 6: Effects of oblateness and $L_{5}$ on the critical mass value for HD98800 system with $\mu=0.45344, q_{1}=0.405$, and $q_{2}=$ 0.205

The positions and stability of libration points in the framework of the circular restricted three-body problem for the systems; Luyten726-8 and HD98800 are described in equations 1-4, 15 \& 16, 26 \& 28. Our results agree with Hassan et al. (2013), in absence of radiations of both primaries and oblateness of the smaller primary. As shown in Tables $1 \& 2$, the positions of the libration points and the critical mass values are affected by the radiations and oblateness of the stated problem and the binary systems. Tables $3 \& 4$ show the effects of the parameters involved on the positions and stability of the libration points $\left(L_{4,5}\right)$. These effects of the parameters shown in Tables $1 \& 2$ are graphically represented in Figures 1-6. The position of the third body lies in the $x z$-plane almost directly above and below the center of the oblate primary.
Considering the range of stability and instability, that is $0<\mu \leq$ $\mu_{c}$ and $\mu_{c}<\mu \leq \frac{1}{2}$ for the libration points been stable and unstable respectively, the libration points are stable and unstable for the systems HD98800 and Luyten 762-8 as it is evidence in Table 2. As presented in Tables $3 \& 4$, all the roots are real, and for each set of values, there exist at least a positive real part and hence in the Lyapunov sense, the stability of the libration points cannot be realized for the systems HD98800 and Luyten 762-8.

\section{CONCLUSION}

In this study, we consider the positions and stability of libration points in the circular restricted problem of three bodies for the systems: Luyten726-8 and HD98800. The results indicate that the libration points are stable and unstable for HD98800 and Luyten 762-8 systems in the range of $0<\mu \leq \mu_{c}$ and $\mu_{c}<\mu \leq$ 
$\frac{1}{2}$ respectively. It was also observed in the Lyapunov sense that the stability of the libration points could not be realized for the systems HD98800 and Luyten 762-8 as all the roots were real and the existence of at least a positive real part for each set of values.

\section{REFERENCES}

AbdulRaheem, A. and Singh J. (2006). Combined effects of perturbations, radiation and oblateness on the stability of libration points in the restricted three-body problem.Astronomical journal. 131: 1880-1885

Abouelmagd, E. I. (2012). Existence and stability of triangular points in the restricted three-body problem. Astrophys. Space Sci. 342:45-53

Abouelmagd, E. I. and El-Shaboury, S.M. (2012), periodic orbits under combined effects of oblateness and radiation in the restricted problem of three bodies. Astrophysics and Space Science, Vol. 341: 331-341

Douskos, C.N., and Markellos, V.V., (2006). "Out-of-Plane equilibrium points in the restricted three-body problem with oblateness". Astronomy and Astrophysics, Vol. 466: 357-362.

Hassan, M. R, Antia, H. M. and Bhatnagar, K. B. (2013), Position and velocity sensitivities at the triangular libration points in the restricted problem of three bodies when the bigger primary is an oblate body. Astrophysics and Space Science, Vol. 346:71-78

Sharma, R. K., (1982). "Linear Stability of triangular points in the generalized photogravitational restricted problem of three bodies", In Sun and Planetary System.(edited by Fricke, W., and Teleki, G.), Dordrecht: Riedel, 435.

Singh, J., and Ishwar, B. (1999). Stability of triangular points in the generalized photogravitational restricted three-body problem.Bull Astronomy Soc India. 27: 415-424

Singh, J. and Leke, O. (2013), "Effects of oblateness, perturbations, radiation and varying masses on the stability of equilibrium points in the restricted three-body problem" Astrophysics and Space Science, Vol. 344:51
Singh, J., Leke, O., (2014). Analytic and numerical treatment of motion of dust grain particle around triangular equilibrium points with post-AGB binary star and disc. Advances in Space research. 54: 1659-1677.

Singh, J. and Taura, J.J. (2013), "Motion in the generalized restricted three-body problem" Astrophysics and Space Science, Vol. 343: 95-106.

Singh, J. and Begha, J.M. (2011), Periodic orbits in the generalized perturbed Restricted three-body problem. Astrophysics and Space Science, Vol. 332: 319-324

Singh, J., Umar, A., (2012). On the stability of triangular equilibrium points in the elliptic R3BP under radiating and oblate primaries. Astrophys. Space Sci. 341: 349-358.

Singh, J., Umar, A., (2013). On out of plane equilibrium points in the Elliptic restricted three-body problem with radiation and oblate primaries. Astrophys. Space Sci. 344: 13-19

Singh J., Amuda T.O., (2015). Out-of-Plane equilibrium points in the photogravitational circular restricted three-body problem with oblateness and P-R Drag. Astronomy and Astrophysics 36:291-305

Singh J., Tyokyaa K.R., (2016). Stability of triangular points in the elliptic restricted three- body problem with oblateness up to zonal harmonic $\mathrm{J}_{4}$ of both primaries. Eur. Phys. J. Plus, 131:365

Singh J., Tyokyaa K.R., (2017). Stability of collinear points in the elliptic restricted three- body problem with oblateness up to zonal harmonic $\mathrm{J}_{4}$ of both primaries. Eur. Phys. J. Plus, 132:330

Szebehely, V.G.: Theory of Orbits. Academic Press, New York (1967)

Wintner, A. (1941).The Analytical foundations of Celestial Mechanics (Princeton university press, Princeton New Jersey). $372-373$.

(C)2020 This is an Open Access article distributed under the terms of the Creative Commons Attribution 4.0 International license viewed via https://creativecommons.org/licenses/by/4.0/ which permits unrestricted use, distribution, and reproduction in any medium, provided the original work is cited appropriately. 